\title{
Rolling Bearing Fault Diagnosis based on Time-frequency Feature Parameters and Wavelet Neural Network
}

\author{
Wang Xing $^{1}$ Qi Xiangdong ${ }^{1}$ and Li Baojin ${ }^{2}$ \\ ${ }^{1}$ Taiyuan University of Science and Technology, Taiyuan Shanxi, 030024, China \\ ${ }^{2}$ Taiyuan Tong xin de Tech. \& Trading Co., Ltd, Taiyuan Shanxi, 030024, China \\ wangxing3969@126.com
}

\begin{abstract}
In this paper, we present a novel method in fault diagnosis of rolling bearing based on time-frequency feature parameters and wavelet neural network. First, the time feature parameters are extracted from the vibration signal. Then the empirical mode decomposition (EMD) is used to decompose the signals of rolling bearings into a number of intrinsic mode functions (IMFs), and then the IMF energy-torques could be calculated through the vibration signal. Finally, those time-frequency feature parameters are taken as fault samples to train wavelet neural network (WNN). The analysis results from the experimental show that the time-frequency feature parameters and WNN is effective in rolling bearing fault diagnosis. This paper provides the theoretical foundation for fault diagnosis in rotary machines.
\end{abstract}

Keywords: wavelet neural network, time-frequency feature parameters, EMD, IMFs, rolling bearing, fault diagnosis

\section{Introduction}

The rolling bearings are the most widely used mechanical components in industrial equipments such as cutting machines, motors, electrical generators and turbo-pumps etc. Detecting the bearing defects at an early stage while the machine is still on operation can help to avoid abnormal event progression and reduce productivity loss [1].

Conventional methods include peak amplitude, root-mean-square amplitude, crest factor, kurtosis, fast Fourier spectra time waveform, sum and difference frequencies analysis and envelope spectra technique, which have proved to be effective in fault diagnosis and are now well established [2-7]. However, when bearing defects occur, the vibration signals often display non-stationary and non-linear behaviours. Therefore, short time Fourier transform (STFT), Wigner-Ville distribution (WVD), empirical mode decomposition (EMD) and wavelet neural network (WNN), have been developed and applied to detect the faults of bearing. In this paper, we proposes the time-frequency feature parameters and wavelet neural network algorithm for the diagnosis of rolling bearings. The practice example shows that the train wavelet neural network can diagnose rolling bearing faults. It provides the theoretical foundation for fault diagnosis in rotary machines, and this method is effective and feasible.

\section{Time-domain Feature Extraction}

The kurtosis factor, margin factor and pulse factor are calculated [8]. They are defined as follows:

Kurtosis factor 
(1)

$$
K_{v}=\frac{\sum_{i=1}^{n} x_{i}^{4}}{n x_{r m s}^{4}}
$$

Margin factor

(2)

$$
C L_{f}=\frac{x_{p e a k}}{x_{r}}
$$

Pulse factor

$$
I_{f}=\frac{x_{p e a k}}{|\bar{x}|}
$$

Where $x_{i}$ is $i$ th sampling point of the signal $x, n$ is the number of points in the signal, $x_{r m s}$ is the root mean square of the signal, $x_{r}$ is the square root of amplitude of the signal, and $|\bar{x}|$ is the absolute average of the signal.

\section{EMD Algorithm and Time-frequency Domain Feature Extraction}

\subsection{EMD Algorithm}

EMD method is able to decompose a signal into some IMFs and one residue. Frequency components contained in each IMF not only relate to the sampling frequency, but also change with the signal itself. Therefore EMD is a self-adaptive signal processing method that can be applied to non-linear and non-stationary process perfectly [9].

Each signal could be decomposed into a number of IMFs, each of which must satisfy the following definition [10]:

(1) In the whole data set, the number of extrema and the number of zero-crossings must either equal or differ at most by one.

(2) At any point, the mean value of the envelope defined by local maxima and the envelope defined by the local minima is zero.

After a series of calculation, the original signals are decomposed as follows:

$$
x(t)=\sum_{i=1}^{n} c_{i}(t)+r_{n}(t)
$$

(4)

where $x(t)$ is the original signals, and $c_{1}(t), c_{2}(t), \mathrm{L}, c_{n}(t)$ are the $1-\mathrm{nth}$ IMFs respectively, while $r_{n}(t)$ is the residue.

\subsection{Time-frequency Domain Feature Extraction}

The steps of time-frequency domain feature extraction are as follows:

(1) The vibration signals are decomposed into some IMFs by using the EMD method, the first $n \operatorname{IMFs} c_{i}(t), \quad i=1,2,3, \mathrm{~L}, n$, which include the most dominant fault energy are chosen to extract the feature.

(2) Calculate the energy-torque of every small time block

The IMF energy-torque is calculated as the following equation: 


$$
E_{i}=\int_{-\infty}^{+\infty} t\left|c_{i}(t)\right|^{2} d t
$$

For discrete signals, the energy-torque is calculated as the following equation:

$$
E_{i}=\sum_{k=1}^{m}(k \cdot \Delta t)\left|c_{i}(k \cdot \Delta t)\right|^{2}
$$

(6)

Where $m$ is the total number of sampling points, $k$ is the sampling points, $\Delta t$ is the sampling period. Calculating the energy-torque $E_{1}, E_{2}$, L for each chosen IMF based on the formula (6).

(3) Constructing the feature vector $T$ in the elements of the energy-torque.

$$
T=\left[\begin{array}{llll}
E_{1} & E_{2} & \cdots & E_{n}
\end{array}\right]
$$

When the energy-torque is a larger numerical, normalizing $T$ and get the normalized feature vector $T^{\prime}$

Among them:

$$
E=\left(\sum_{i=1}^{n}\left|E_{i}\right|^{2}\right)^{\frac{1}{2}}
$$

(8)

(9)

The IMF energy-torque is calculated as the following equation [11]:

$$
E_{i}=\int_{-\infty}^{+\infty}\left|c_{i}(t)\right|^{2} d t
$$

\section{Wavelet Neural Network Model}

Wavelet neural network is a feed forward neural network on the basis of wavelet transform that combines the time-frequency localization of wavelet transform and selflearning function of neural network. It takes wavelet space as feature space of pattern recognition and realizes the feature extraction of the signal by weighting the inner product of wavelets base and signal vector, which can efficiently learn the input/output characteristics of the system without too much prior information such as structure, characteristics and so on [12].

The WNN consists of three layers: input layer, hidden layer and output layer. The connections between input units and hidden units, and between hidden units and output units are called weights $w_{t i}$ and $W_{t}$, respectively. The proposed WNN architecture is shown in Fig.1.

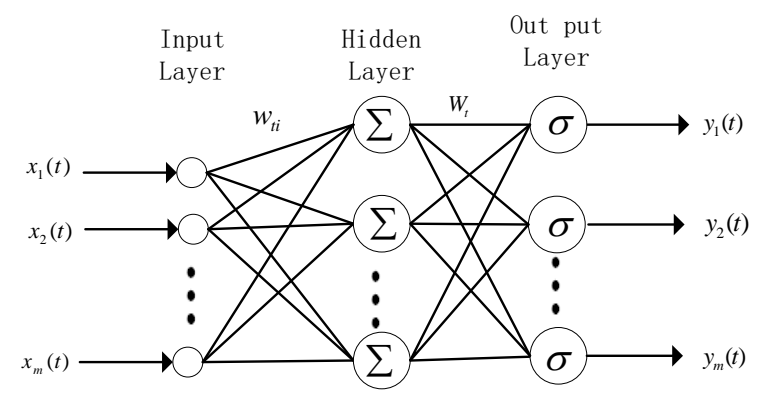

Figure 1. Topology Structure of Multi-layer Wavelet Neural Network 
In this WNN, the training procedure is described as follows [13]:

Initialising the dilation parameter $a_{t}$, translation parameter $b_{t}$ and node connection weights $w_{t i}, W_{t}$ to some random values. All those random values are limited in the interval $(0,1)$.

Input data $X_{n}(i)$ and the corresponding output values $V_{n}^{T}$, where $i$ varies from 1 to $S$, representing the number of the input nodes, $n$ represents the $n$th data sample of training set, and $T$ represents the target output state.

The output value of the sample $V_{n}$ is calculated with the following formula:

$$
V_{n}=\sum_{t=1}^{T} W_{t} \psi\left(\frac{\sum_{i=1}^{S} w_{t i} x_{n}(i)-b_{t}}{a_{t}}\right)
$$

where $\psi$ is considered a mother wavelet, such as the Morlet wavelet filter, and is represented by

$$
\psi(t)=\cos \left(\omega_{0} t\right) \exp \left(-0.5 t^{2}\right)
$$

To reduce the error, $w_{t i}, W_{t}, a_{t}, b_{t}$ are adjusted using $\Delta w_{t i}, \Delta W_{t}, \Delta a_{t}, \Delta b_{t}$. In the WNN, the gradient descend algorithm is employed, through the following equations,

$$
\Delta w_{t}(j+1)=-\eta \frac{\partial E}{\partial w_{t}(j)}+\alpha \Delta w_{t}(j)
$$

$$
\Delta W_{t}(j+1)=-\eta \frac{\partial E}{\partial W_{t}(j)}+\alpha \Delta W_{t}(j)
$$

$$
\Delta a_{t}(j+1)=-\eta \frac{\partial E}{\partial a_{t}(j)}+\alpha \Delta a_{t}(j)
$$

$$
\Delta b_{t}(j+1)=-\eta \frac{\partial E}{\partial b_{t}(j)}+\alpha \Delta b_{t}(j)
$$

where the error function $E$ is taken as:

$$
E=\frac{1}{2} \sum_{n=1}^{N}\left(V_{n}^{T}-V_{n}\right)^{2}
$$

and, $N$ standing for the data number of training set, $\eta$ and $\alpha$ being the learning rate and the momentum term, respectively.

The process continues until $E$ satisfies the given error criteria, and the whole training of the WNN is completed.

\section{Experimental Results and Analysis}

To verify the validity of the proposed method, three kinds of fault types are researched under no-load condition: (1) rolling fault vibration signal, (2) outer ring fault vibration signal, (3) inner ring fault vibration signal. 


\subsection{The Process of Time-frequency Domain Feature Extraction}

The time-domain feature parameters are calculated from the original vibration signal. The outer ring fault vibration signal is shown in Fig.2. The vibration signal is decomposed by EMD and the result is shown in Fig.3, and then the IMF energy-torques could be calculated through the vibration signal. The time-frequency feature parameters are divided into two groups, namely, the training group and the testing group. The training group is in table 1 . The testing group is in table 2 . The WNN is used to diagnose the faults; the inputs and outputs of the WNN are all memberships concretely, the inputs are the memberships of the kurtosis factor, margin factor, pulse factor and IMFs energy-torque on each characteristic domain, and the outputs are memberships of each fault.

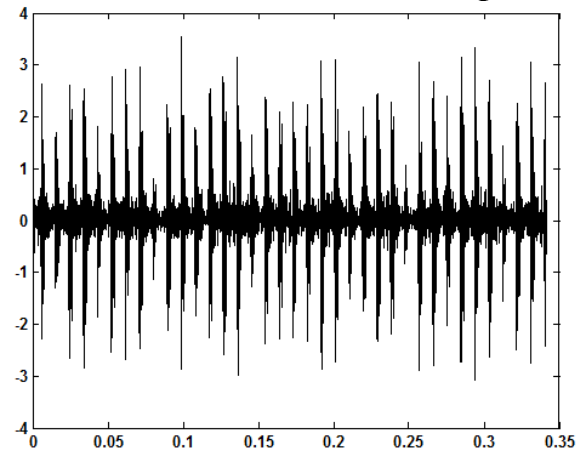

Figure 2. The Time Domain of the Outer Ring Fault Signal

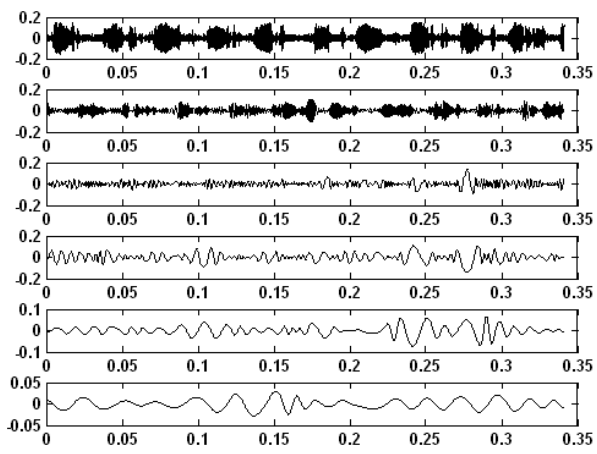

Figure 3. Decomposition of the Outer Ring Fault Signal into Intrinsic Mode Functions

$\begin{aligned} & \text { kurtosis margin } \\ & \text { factor }\end{aligned}$ factor
factor

Table 1. Sample Data of Bearing Operation

\begin{tabular}{llllllllll}
\hline $\begin{array}{l}\text { kurtosis margin pulse } \\
\text { factor }\end{array}$ & $E_{1}$ & $E_{2}$ & $E_{3}$ & $E_{4}$ & $E_{5}$ & Fault status & $\begin{array}{c}\text { Fault } \\
\text { vector }\end{array}$ \\
\hline 4.2623 & 7.3444 & 6.1675 & 0.2122 & 0.7033 & 0.6023 & 0.3104 & 0.0358 & rolling fault signal & $\left(\begin{array}{llllll}1 & 0 & 0\end{array}\right)$ \\
24.1784 & 32.34 & 20.6237 & 0.8381 & 0.5077 & 0.1588 & 0.0929 & 0.077 & outer ring fault signal & $\left(\begin{array}{lllll}0 & 1 & 0\end{array}\right)$ \\
16.9063 & 37.442 & 18.759 & 0.9531 & 0.1253 & 0.2522 & 0.0437 & 0.1015 & inner ring fault signal & $\left(\begin{array}{llll}0 & 0 & 1\end{array}\right)$ \\
\hline
\end{tabular}

Table 2. Testing Data 


\subsection{The Process of WNN}

The WNN used for fault diagnosis consist of three layers: an input layer, a hidden layer and an output layer. The WNN architecture is 8-5-3.

\begin{tabular}{|c|c|c|c|}
\hline Fault status & Ideal outputs & Actual outputs & Testing results \\
\hline rolling fault signal & $\left(\begin{array}{lll}1 & 0 & 0\end{array}\right)$ & $(1.0215-0.16050 .0012)$ & rolling fault signal \\
\hline outer ring fault signal & $\left(\begin{array}{lll}0 & 1 & 0\end{array}\right)$ & $(0.10161 .02540 .0055)$ & outer ring fault signal \\
\hline inner ring fault signal & $\left(\begin{array}{lll}0 & 0 & 1\end{array}\right)$ & $(0.0117-0.10591 .0036)$ & inner ring fault signal \\
\hline
\end{tabular}

\section{Table 3. Testing Results of WNN}

Table 3 shows the testing results of the WNN. As shown in this table, the classification of WNN are all close to the corresponding ideal outputs of the examination sample, the results obtained using WNN not only does the high capabilities of this algorithm prove in order to find the optimal solution but also verifies the effectiveness of the time-frequency feature parameters for the algorithm presented in this paper.

\section{Conclusion}

In this paper, a method for rolling bearing fault diagnosis is presented based on time-frequency feature parameters and wavelet neural network. The EMD is used to decompose the original vibration signal, and the time-frequency feature parameters are extracted, then those feature parameters are taken to train WNN. The experimental results indicate that the proposed method can extract the feature and classify the different faults of rolling bearing. The future work will be focused on the application of condition monitoring and fault diagnosis in other rotation machines.

\section{Acknowledgement}

This paper was supported by the International Science \& Technology Cooperation Program of China (2014DFR70280).

\section{References}

[1] P. M. Chun and T. W. Chang, "Using appropriate IMFs for envelope analysis in multiple fault diagnosis of ball bearings", International Journal of Mechanical Sciences, vol. 69, (2013), pp. 114-124.

[2] J. Ma and C. J. Li, "Detection of localized defects in rolling element bearings via composite hypothesis test", Symposium on Mechatronics, DSV-V01, American Society of Mechanical Engineers, (1993).

[3] H. R. Martin, "Statistical moment analysis as a means of surface damage detection", Proceedings of the International Modal Analysis Conference, (1989).

[4] E. Volker and H. R. Matin, "Application of Kurtosis to Damage Mapping", Proceedings of the International Modal Analysis Conference, (1986).

[5] G. A. Radcliff, "Condition monitoring of rolling element bearings using the enveloping technique", Machine Condition Monitoring, Mechanical Engineering Publication Ltd, (1990), pp. 55-67.

[6] W. Peter, Y. H. Tse and R. Y. Peng, "Wavelet analysis and envelope detection for rolling element bearing for rolling element bearing fault diagnosis-their affectivities and flexibilities", Journal of Vibration and Acoustics, vol. 123, (2001), pp. 303-310.

[7] G. T. Zheng and W. J. Wang, "A new cesptral analysis procedure of recovering excitations for transient components of vibration signals and applications to rotating machinery condition monitoring", Journal of Vibration and Acoustics, vol. 123, (2001), pp. 222-229.

[8] L. Y. Guo, M. J. Zuo and H. Z. Jia, "A multidimensional hybrid intelligent method for gear fault diagnosis”, Expert Systems with Applications, vol. 37, (2010), pp. 1419-1430. 
[9] S. Z. Jieand C. X. Feng, "A novel intelligent gear fault diagnosis model based on EMD and multi-class TSVM", Measurement, vol. 45, (2012), pp. 30-40.

[10] J. Huang, H. X. Guang and X. Geng, "An intelligent fault diagnosis method of high voltage circuit breaker based on improved EMD energy entropy and multi-class support vector machine", Electric Power Systems Research, vol. 81, (2011), pp. 400-407.

[11] G. F. Bin, J. J. Gao and X. J. Li, "Early fault diagnosis of rotating machinery based on wavelet packetsEmpirical mode decomposition feature extraction and neural network", Mechanical Systems and Signal Processing, (2012), pp. 696-711.

[12] M. Amina and V. S. Kodogiannis, "Identification of the Listeria monocytogenes survival curves in UHT whole milk utilising local linear wavelet neural networks", Expert Systems with Applications, vol. 39, (2012), pp. 1435-1450.

[13] M. Y. Boa and Z. J. Hua, "Research on WNN aero dynamic modeling from flight data based on improved PSO algorithm”, Neurocomputing, vol. 83, (2012), pp. 212-221.

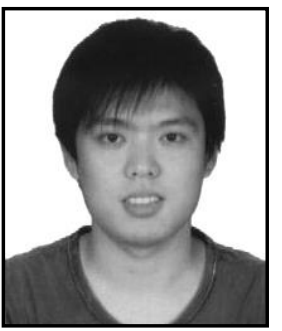

Xing Wang, he received his B.E degree from Taiyuan University of Science and Technology, in electronic information engineering, China, in 2004, and the M.E degree from Taiyuan University of Science and Technology, in power electronics and power drives, China, in 2007. Since 2007, he is working at Taiyuan University of Science and Technology, Taiyuan, China. His research interests include the development of railway wagon testing equipment and the design of computer intelligent detection software. 
International Journal of Control and Automation Vol. 8, No.4 (2015) 